\title{
Drug-induced ocular cicatricial pemphigoid: a series of clinico- pathological reports
}

\begin{abstract}
Ocular cicatricial pemphigoid is a slowly progressive disease of mucous membranes and skin of unknown but presumed autoimmune aetiology. We describe eight cases of presumed drug-induced cicatrising conjunctival changes simulating ocular cicatricial pemphigoid, following the chronic use of topical glaucoma medication. In three of four patients who underwent conjunctival biopsy of the inferior fornix, this revealed histopathological changes similar to ocular cicatricial pemphigoid.
\end{abstract}

Key words Conjunctiva, Inflammation, Pemphigoid, Pseudopemphigoid, Symblepharon

Ocular cicatricial pemphigoid (OCP) is an inflammatory and cicatrising disease of the conjunctiva and other mucous membranes that causes severe visual handicap in up to one-third of patients. ${ }^{1}$ Skin lesions are extremely rare. It is usually bilateral. Clinical features include medial canthal keratinisation and obliteration, progressive shortening of the fornices, cicatricial entropion with trichiasis, development of symblepharon or total ankyloblepharon in severe cases, keratinisation of the ocular surface, xerosis and frequent corneal complications including corneal ulcers and pannus formation.

The disease is characterised by the abnormal binding of immunoglobulins to the basement membranes of the skin and mucous membranes. ${ }^{2}$ Acutely, this causes complement activation and infiltration of the conjunctiva by neutrophils, macrophages and activated $\mathrm{T}$ lymphocytes. ${ }^{3}$ The conjunctival and corneal scarring are a consequence of this. Once this has occurred, the prognosis for visual rehabilitation is poor and, therefore, the prevention of disease progression is important. ${ }^{4}$

We report eight cases of probable druginduced OCP with conjunctival biopsy confirming this in three of four cases. Two of the patients had unilateral cicatricial changes due to unilateral usage of topical glaucoma therapy.

\section{Materials and methods}

All eight patients were recruited as outpatients and presented with lid entropion/trichiasis, uncontrolled glaucoma or corneal problems. Four of the seven patients had inferior conjunctival fornix biopsies in the area of symblepharon formation. Informed consent was obtained from all patients prior to the conjunctival biopsy. The specimens were sent fresh to the pathology department, sandwiched between two moist strolls, and were examined immediately.

\section{Immunofluorescence studies}

The conjunctiva was snap frozen in an isopentane slurry. Four-micrometre cryostat sections were cut and fixed in acetone for $10 \mathrm{~min}$. The sections were washed in phosphate-buffered saline (PBS) for $5 \mathrm{~min}$, then one drop of the fluorescein-labelled antibodies was placed onto the slide and incubated at room temperature in the dark for $30 \mathrm{~min}$. After rinsing and washing in PBS for $5 \mathrm{~min}$, the slides were then mounted in 50/50 glycerol/PBS. Sections were viewed under a fluorescence microscope. Antisera used were fluoresceinlabelled anti-human globulin, IgM, IgA, IgM, fibrin, C3, C1q, albumin and anti-rabbit globulin (all Silenus reagents). The rest of the specimen was defrosted and processed in paraffin wax for $H \& E$ sections and periodic acid-Schiff (PAS) staining for goblet cells.

\section{Case histories}

\section{Case 1}

An 86-year-old man was diagnosed as having pseudoexfoliation glaucoma in 1987. He was commenced on triple therapy of pilocarpine, dipivefrine and timolol eyedrops for adequate control of his intraocular pressures in both eyes. He had a right cataract extraction in 1989, which was complicated by posterior capsule rupture, and necessitated a vitrectomy. He developed corneal oedema post-operatively that did not settle. In 1990 he had a right corneal transplant for pain relief. Past medical history revealed he

\author{
Z Butt \\ A. MCNab \\ Departrnent of \\ Ophthalmology \\ Royal Victorian Eye and Ear \\ Hospital \\ Melbourne \\ Australia \\ D. Kaufrnan \\ P. McKelvie \\ Department of Pathology \\ St Vincent's Hospital \\ Metbourne \\ Australia \\ Z. Butt \\ Queen Elizabeth Hospital \\ Gayton Road \\ King's Lynn \\ Norfolk \\ UK
}




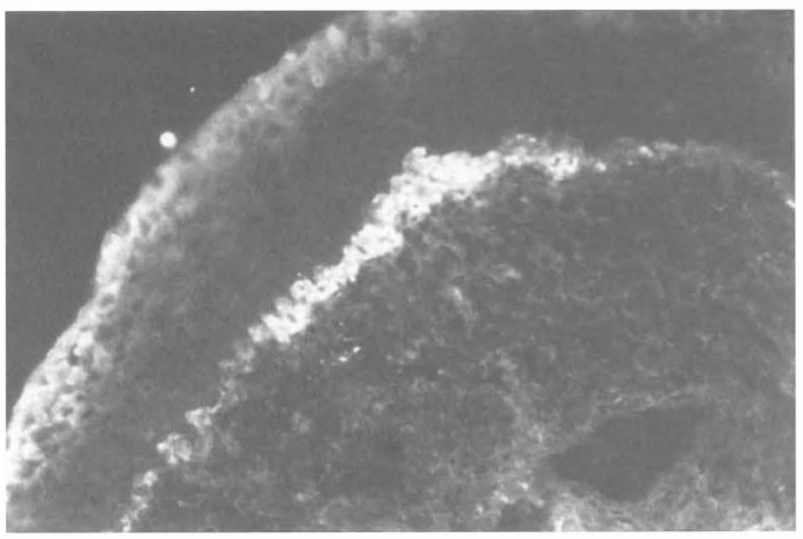

Fig. 1. Case 1. Inmunofluorescence photograph of positive linear C3 fluorescent staining at the basement membrane zone $(\times 450)$.

had ischaemic heart disease and had been a non-insulindependent diabetic for 15 years, on oral hypoglycaemic drugs.

In 1995, he presented with a foreign body sensation in his eyes. On examination he was noted to have a right medial canthal keratinisation, marked cicatrising conjunctival changes worse in the right than left eye, subconjunctival tarsal scarring, and right upper and lower lid cicatricial entropion with trichiasis. Visual acuity was no perception of light right and 6/9 corrected left.

He underwent a right upper lid anterior lamellar reposition and mucous membrane graft, and a right lower lid retractor tightening that settled his trichiasis. He also underwent a right inferior conjunctival fornix biopsy in the area with maximal cicatricial changes.

\section{Pathology}

The conjunctival biopsy showed acanthosis with parakeratosis and scattered dyskeratotic cells throughout the epithelium. No goblet cells were noted. There was some subepithelial scarring and a dense stromal infiltrate of mononuclear cells. Immunofluorescence studies showed strong linear $\mathrm{C} 3$ reactivity along the basement membrane zone (Fig. 1), but $\mathrm{C} 1 \mathrm{q}$ and immunoglobulins were negative.

\section{Case 2}

A 75-year-old woman presented to the oculoplastics department with recurrent bilateral lower lid trichiasis. Electrolysis on two previous occasions had failed to eradicate the trichiasis. She therefore had left lower lid everting sutures later on, which resolved the problem.

She had open angle glaucoma that had been diagnosed 10 years previously and was using timolol and dipivefrine eyedrops in both eyes. She also had known age-related macular degeneration in her left eye and a history of dry eyes for which she used artificial tears as required. She was hypertensive and was on oral medication of ramipril, frusemide, aspirin and simvastatin.
On examination, corrected visual acuity was $6 / 6$ right and 6/12 left with a cup/disc ratio of 0.85 in both eyes. She was noted to have bilateral medial canthal keratinisation and right inferior conjunctival symblepharon. A right inferior conjunctival fornix biopsy in 1996 was positive for markers of OCP.

She was commenced on oral dapsone $25 \mathrm{mg}$, increasing to $50 \mathrm{mg}$ after a fortnight, which only partially relieved her symptoms.

\section{Pathology}

The conjunctival biopsy showed mild parakeratosis with almost total depletion of goblet cells. Moderate focal infiltrates of small lymphocytes and plasma cells were noted in the stroma. Immunofluorescence studies showed weak patchy reactivity for $\mathrm{C} 3$ along the basement membrane zone, but $\mathrm{C} 1 \mathrm{q}$ and immunoglobulins were negative.

\section{Case 3}

A 63-year-old woman was seen at the oculoplastics department in 1994 with biopsy-proven OCP. She had a long history of open angle glaucoma that had been treated with pilocarpine and dipivefrine for 7 years. Intraocular pressures were not adequately controlled and she underwent bilateral trabeculectomy and cataract extraction with intraocular lens implantation. However, these failed and it was only subsequently realised that conjunctival cicatrisation may have accounted for this. She had previously been diagnosed with systemic lupus erythematus, Sjögren's syndrome and ischaemic heart disease. She was on oral medication of captopril, aspirin and frusemide.

On examination, corrected visual acuity was $6 / 36$ right and counting fingers vision left. There was advanced glaucomatous optic atrophy with a cup/disc ratio of 0.9 , and correspondingly severely constricted visual fields. She had bilateral severe conjunctival cicatricial changes in both eyes with symblepharon formation. This was felt to be related to her glaucoma therapy, which was therefore changed to oral acetazolamide and betaxolol $0.5 \%$ eyedrops to both eyes. She was also commenced on Lacri-Lube (Allergan) ointment for dry eyes and dapsone $50 \mathrm{mg}$ for several months with relief of symptoms. An inferior conjunctival biopsy in 1994 confirmed OCP.

\section{Case 4}

A 72-year-old man had a history of open angle glaucoma for which he had been on topical pilocarpine for 10 years, topical dipivefrine for 5 years and betaxolol for 3 years. He had a 2 year history of bilateral lower lid trichiasis. His general health was good and he was on no other medication.

On examination, corrected visual acuity was 6/9 right and 6/6 left. He had subconjunctival scarring worse on the right with shallowing of the inferior fornices and 


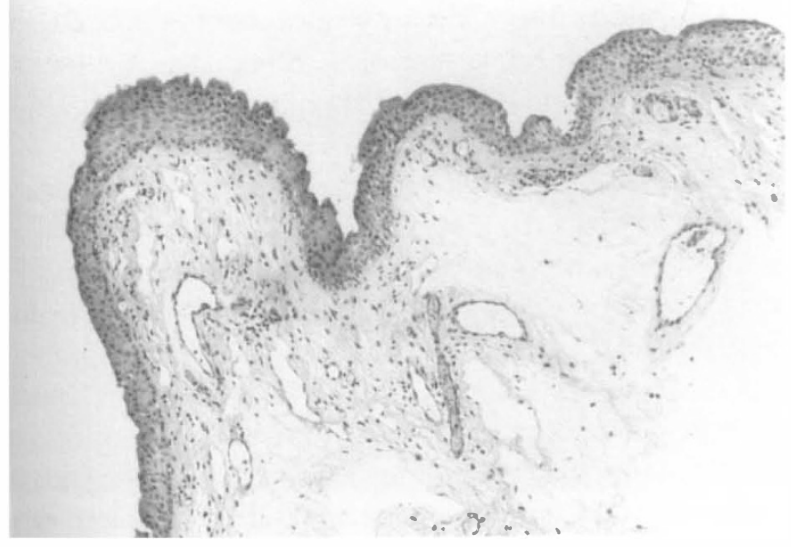

Fig. 2. Case' 4. Photomicrograph of comjunction showing a relatiocly normal eprithelium and focal lymuhoplasmacytic infiltrates in the' stroma (HE,E, $\times .5()())$.

early symblepharon. Both medial fornices were slightly reduced. The upper tarsus was normal. Three

conjunctival biopsy specimens were examined and were all negative for OCP markers. He had bilateral lower lid cryotherapy in 1995 which settled his trichiasis.

\section{Pathology}

The right conjunctival biopsy showed an almost normal complement of goblet cells in the epithelium and mild focal lymphoplasmacytic infiltrates in the stroma (Fig. 2). Immunofluorescence studies were negative.

\section{Case 5}

A 75-year-old man complained of epiphora in both eyes when seen in May 1996. He had previously had bilateral cataract and intraocular lens implantation. He had also had unsuccessful bilateral trabeculectomies. The left cataract surgery, performed in India, was complicated, and led to blindness. He had been on topical isoptocarpine and betaxolol since 1989, when he was diagnosed with glaucoma. In 1993, due to uncontrolled intraocular pressures, dipivefrine was commenced in both eyes for several years and daily oral acetazolamide $250 \mathrm{mg}$. His general health was good and he was on no other oral medication.

On examination, corrected visual acuity was $6 / 9$ right and no perception of light left. He had bilaterally shortened inferior fornices with subconjunctival scarring This was presumed to be drug-induced OCP secondary to topical glaucoma therapy. He did not undergo a conjunctival biopsy.

\section{Case 6}

A 76-year-old woman had been diagnosed with open angle glaucoma 20 years previously, for which she had been on triple therapy of topical dipivefrine, pilocarpine and timolol and oral acetazolamide $125 \mathrm{mg}$ twice daily. She had a strong family history of glaucoma. She also had hypercholesterolaemia, for which she was on oral simvastatin. She had been troubled with trichiasis in both eyes for the past year and had been epilating her lashes regularly. On examination she had a corrected visual acuity of 6/6 right and 6/12 left with a cup/disc ratio of 0.9 in both eyes. She had bilateral moderate conjunctival cicatrisation, especially over the medial canthus. She also had a band of upper lid tarsal scarring leading to upper lid cicatricial entropion with trichiasis. She had surgical treatment for the bilateral entropion with anterior lamellar reposition with good results. Her glaucoma was not adequately controlled on all her treatment and she therefore underwent bilateral trabeculectomy with 5-fluorouracil surgery, with successfully controlled pressures. She did not undergo a conjunctival biopsy.

\section{Case 7}

This 67-year-old man had suffered a penetrating eye injury in 1984, necessitating repair of a full-thickness corneal laceration. He developed peripheral anterior sy'nechiae 4 years later in the quadrant of his injury together with complicated angle recession over $270^{\circ}$. As a consequence, he suffered secondary glaucoma and was commenced on topical timolol to the right eye in 1988. This did not control his intraocular pressure adequately and therefore dipivefrine was added 1 month later but with no success. He therefore underwent right trabeculectomy which controlled his intraocular pressure initially, but it later rose again. He was then commenced again on topical timolol to which was added dipivefrine, pilocarpine and dichlorphenamide $100 \mathrm{mg}$ b.d. orally, as acetazolamide was not tolerated due to side-effects. He had a past medical history of bronchitis and systemic hypertension. He was on oral medication of chlorothiazide and diazepam.

In 1993 he presented with a foreign body sensation in the right eye. On examination, his corrected visual acuity was $6 / 12$ right and 6/6 corrected left. He was noted to have right upper lid entropion with trichiasis due to extensive conjunctival cicatrisation with loss of the fornices and caruncle (Fig. 3). These changes were entirely unilateral with no evidence of conjunctival cicatrisation on the left (Fig. 4). It was therefore felt that

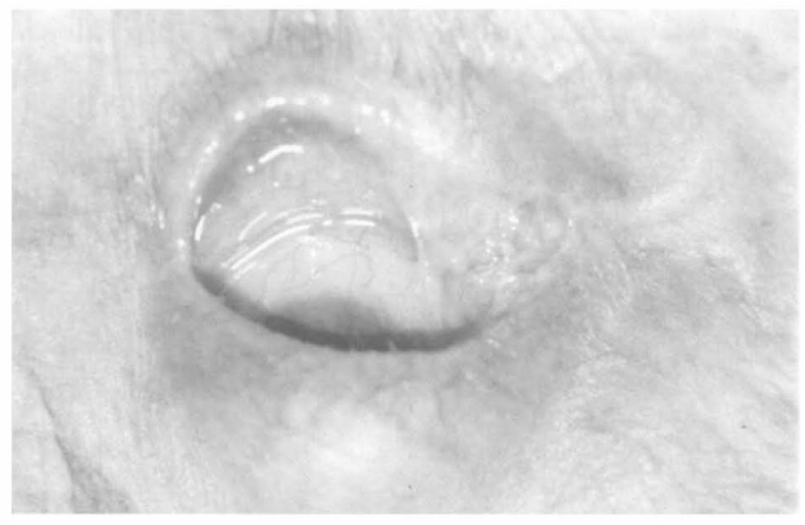

Fig. 3. Case' 7. Right uppere lid entroprion with trichiasis. There' is extensia'e comjunctional cicatrisation ath loss of the formice's and carmucle. 


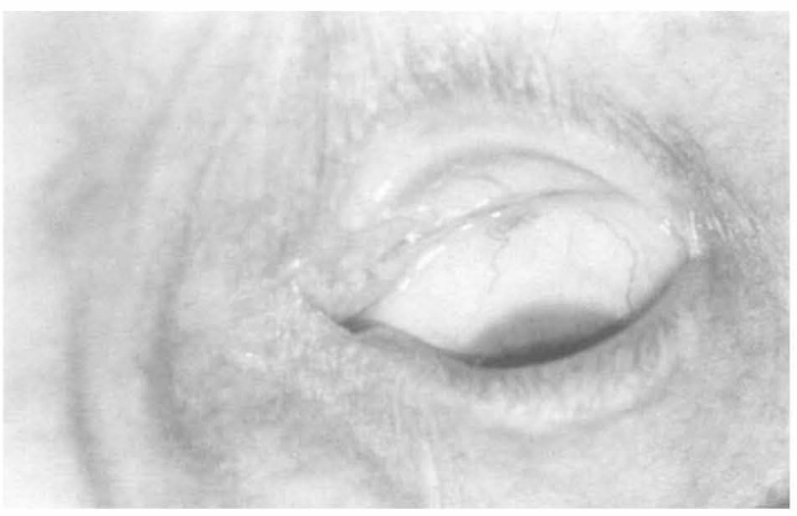

Fig. 4. Case 7. Left eye. There is no evidence of conjunctival cicatrisation.

his symptoms and signs were related to unilateral topical glaucoma therapy and all his treatment was stopped except for topical timolol and oral dichlorphenamide. His intraocular pressures remained at borderline values on this therapy. He had a right upper lid medial lash excision and anterior lamellar reposition in 1995 with resolution of his trichiasis. No conjunctival biopsy was performed.

\section{Case 8}

This 91-year-old woman was seen with entropion and associated trichiasis in her left eye. This was treated with regular epilation after successful cryotherapy twice previously. However, she developed a corneal ulcer as a complication and thereafter was treated with a bandage contact lens, which was changed monthly. She had had cataract surgery with intraocular lens implantation in her left eye 3 years previously. Her right eye had poor vision due to a previous central retinal vein occlusion. She also had open angle glaucoma for which she used dipivefrine, timolol and pilocarpine in her left eye only. She had a history of hypertension and an underactive thyroid for which she was on thyroxine.

On examination, visual acuity was counting fingers right and corrected 6/9 left. She had marked ocular cicatricial changes in her left eye, the right eye being completely normal (Fig. 5). There was medial fornix and

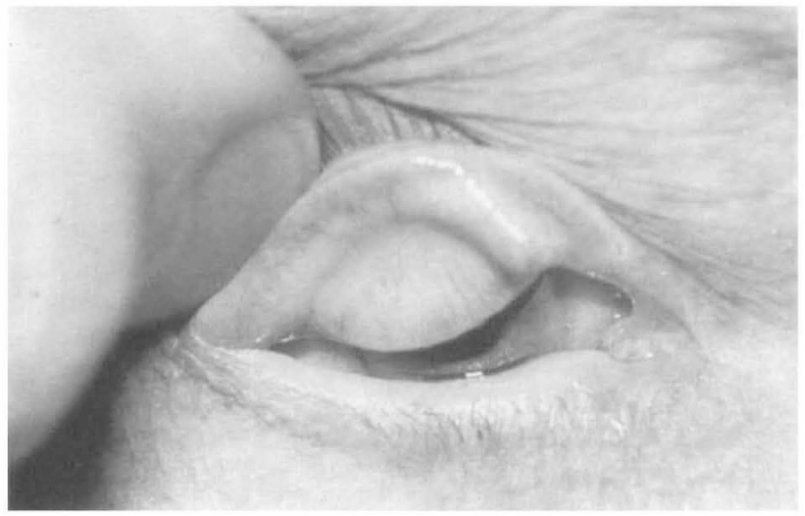

Fig. 5. Case 8. Normal right eye.

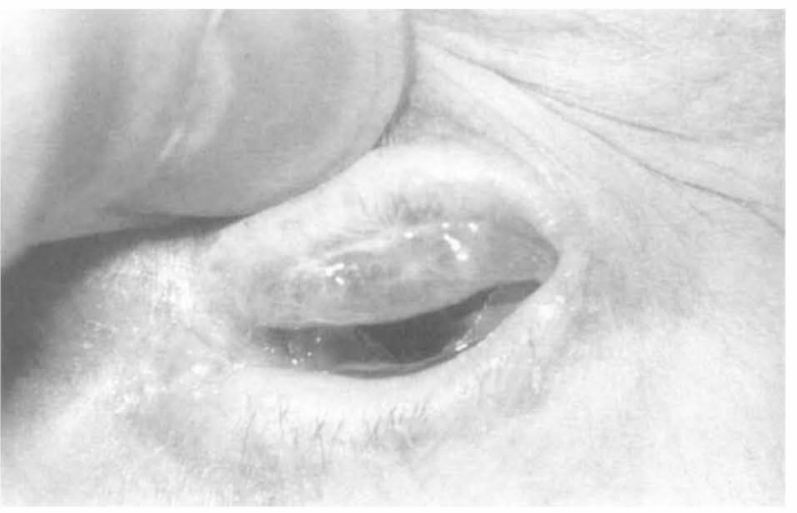

Fig. 6. Case 8. Left eye showing upper lid cicatricial changes.

upper lid cicatricial entropion with trichiasis of her left eye (Figs. 6, 7). She underwent a left terminal tarsal rotation procedure which corrected her left upper lid entropion and trichiasis. She did not have a conjunctival biopsy.

\section{Discussion}

Rare cases of OCP with corneal and conjunctival scarring have occurred in patients using eyedrops containing demecarium bromide, ecothiopate iodide, adrenaline, pilocarpine, dipivefrine or timolol. ${ }^{5-13}$

According to Pouliquen et al., ${ }^{8}$ OCP has been diagnosed and studied microscopically in three patients using topical timolol. In one of these patients, timolol was the only drug being used. OCP has been ascribed in several cases to long-term treatment with adrenaline, with usually a tendency to be arrested or improved when treatment is stopped. ${ }^{8,10,14-16}$ Topical dipivefrine (Propine, Allergan) is an ophthalmic pro-drug of adrenaline used in the treatment of glaucoma and was designed to minimise some of the unwanted effects of adrenaline on the anterior segment of the eye. However, Blanchard ${ }^{10}$ carried out a prospective study of 100 patients with glaucoma and revealed three cases of conjunctival shrinkage and symblepharon. Two of these cases were related to the long-term use of dipivefrine. Chronic topical pilocarpine use has also been implicated

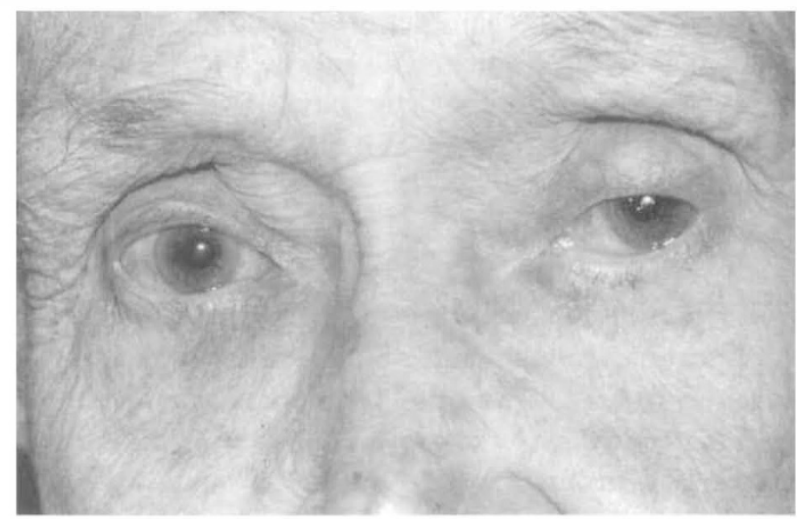

Fig. 7. Case 8. Left upper lid entropion with trichiasis. 
in cicatricial pemphigoid. $0,4,9,11,12$ Cases in which pilocarpine has been used in only one eye and pemphigoid has developed in the same eye have been looked upon as evidence that the condition has been caused by the drug.

Two patients with unilateral OCP who had been using topical ecothiopate iodide (Phospholine Iodide,

Dominion) for 6-9 years have been reported in the literature. ${ }^{13}$ Both cases were studied histologically and immunopathologically in the affected and non-affected eyes. This was the first report of the apparent induction of clinico-pathological changes similar to those of OCP with topically applied medication. Hirst et al. " reported unilateral cicatricial conjunctival changes in a patient who had been on long-term topical demecarium bromide to the affected eye. The unilaterality of the symptoms and signs and the non-progression of signs after cessation of therapy was strong indication of a topical drug-induced disease process.

Practolol is the only oral beta-blocker that can cause ocular signs similar to those in OCP. ${ }^{\top}$ Practolol is a cardioselective beta-adrenergic receptor blocking drug with some intrinsic sympathomimetic activity, which was introduced into clinical use in 1969 but withdrawn in 1975 because of its ocular and other side-effects (retroperitoneal fibrosis).

It is not entirely clear whether topical glaucoma therapy promotes OCP in the first instance or acclerates the emergence of OCP in a predisposed individual. There is no evidence at present to accept or reject either immune or toxic-initiated injury due to long-term topical drug therapy as the cause of drug-induced cicatricial conjunctival disease. Clinically, drug-induced pemphigoid looks identical to OCP and it progresses in the same way, with both categories of patients responding to immunosuppressives. ${ }^{\wedge}$ Also unilateral pemphigoid has been documented before, ${ }^{\phi, 11}$ related to topical therapy in the same eye for unilateral glaucoma. As unilateral OCP is rare, ${ }^{18}$ this shows a strong association between developing the disease and longterm use of glaucoma therapy. This is similar to our cases 7 and 8 with unilateral pemphigoid changes due to glaucoma therapy. The resolution of symptoms on stopping topical therapy ${ }^{8,111,1+16}$ and the apparent induction of clinico-pathological changes similar to those of OCP helps strengthen this association. ${ }^{8.12}$ However, the histopathology in drug-induced pemphigoid can be varied with no set pattern diagnostic of OCP. ${ }^{1(11}$ This is also true of OCP, where immunological testing has produced conflicting and inconsistent results. ${ }^{14.21}$ In our cases 1, 2 and 3 the conjunctival biopsies were positive for OCP markers, suggesting that the disease process was associated with topical glaucoma therapy. In druginduced pemphigoid there is no involvement of any other mucous membrane (apart from conjunctiva) or skin as can occur in OCP, and this is therefore related almost certainly to usage of topical medication, with appearance of the disease process at the site of application. However, Pouliquen et al. ${ }^{.}$observed the appearance of OCP in the untreated eyes of 4 patients with unilateral drug-induced pemphigoid and this is strong evidence that topical glaucoma therapy works by sensitising the predisposed individual to the more rapid emergence of the chronic cicatrising features of $\mathrm{OCP}^{5} .3$

The rational approach to the chronically medicated patient with inflammatory conjunctival signs and symptoms is to discontinue all medications, thereby allowing the initial pathological process and the medication-induced toxicity to resolve by natural mechanisms.

The same approach should apply to glaucoma patients, as there is already clear evidence that stopping the topical glaucoma therapy causes resolution of symptoms and stabilisation of the disease. ${ }^{.10 .1+10}$ If the patient has early signs of drug-induced pemphigoid, early surgical therapy should be the treatment of choice in the form of trabeculectomy rather than alternative topical therapy, because all glaucoma topical medication has shown emergence of pemphigoid." "Even betablocker therapy is not innocuous in this respect." If the disease is allowed to progress, late trabeculectomy surgery will surely fail due to the nature of the scarring process in this disease, as we noted in case 3 . Also, a high index of suspicion and a greater awareness of this condition in glaucoma clinics is absolutely essential to enable predisposed individuals to be identified early in the disease process and treated appropriately. At present, this condition is being underdiagnosed in its early stages due to failure of recognition. This is probably related to not fully appreciating cicatrising changes as a complication of glaucoma therapy and therefore not actively looking for it in each glaucoma patient at every clinic visit, which would be a simple rapid screening measure. Quite often, it comes to attention for the first time when severe complications have developed such as keratinisation of the cornea with pannus formation, corneal ulceration and lid entropion with trichiasis, by which time the disease process has usually progressed a moderate amount and trabeculectomy surgery has a high failure rate. The only alternative in such cases would be to continue topical glaucoma therapy and commence patients on immunosuppressive drugs, which in their own right have unpleasant side-effects. Allowing patients to advance to this stage of the disease could be avoided with a heightened awareness of this disease process in glaucoma patients on topical drug therapy.

We are very grateful to Mr Michael Loughnan for allowing us to utilise his patient records.

\section{References}

1. Hardy KM, Perry HO, Pingree CC. Benign mucous membrane pemphigoid. Arch Dermatol 1971;104:467-75.

2. Mondino BJ, Ross AN, Rabin BS, Brown SI. Autoimmune phenomena in ocular cicatricial pemphigoid. Am J Ophthalmol 1977;83:443-50.

3. Rice BA, Foster CS. Immunopathology of cicatricial pemphigoid affecting the conjunctiva. Ophthalmology 199();97:1476-83. 
4. Foster CS. Cicatricial pemphigoid. Trans Am Ophthalmol Soc $1986 ; 84: 527-663$.

5. Chan LS, Soong HK, Foster CS, et al. Ocular cicatricial pemphigoid occurring as a sequela of Stevens-Johnson syndrome. JAMA 1991;266:1543-6.

6. Fiore PM, Jacobs IH, Goldberg DB. Drug induced pemphigoid. Arch Ophthalmol 1987;105:1660-3.

7. Fiore PM. Drug induced ocular cicatrisation. Int Ophthalmol Clin 1989;29:147-50.

8. Pouliquen Y, Patey A, Foster CS, Goichot L, Savoldelli M. Drug induced cicatricial pemphigoid affecting the conjunctiva: light and electron microscopic features. Ophthalmology 1986;93:775-83.

9. Saraux H, Offret H, DeMimerand EDR. Ocular pseudopemphigus induced by collyria: concerning 3 observations. Bull Soc Ophtalmol Fr 1980;80:41.

10. Blanchard DL. Adrenergic associated symblepharon. Glaucoma 1987;9:18-20.

11. Hirst LW, Werblin T, Novak M, Green WR, Pollack I. Druginduced cicatrizing conjunctivitis simulating ocular pemphigoid. Cornea 1982;1:121-8.

12. Tseng SC, Maumenee AE, Stark WJ, Maumenee IH, Jensen $A D$, Green WR, Kenyon KR. Topical retinoid treatment for various dry eye disorders. Ophthalmology 1985;92:717-27.
13. Patten JT, Cavanagh HD, Allansmith MR. Induced ocular pseudopemphigoid. Am J Ophthalmol 1976;82:272-6.

14. Hoffer KJ. Pemphigoid related to epinephrine treatment [letter]. Am J Ophthalmol 1977;83:601.

15. Kirstensen EB, Norn MS. Benign mucous membrane pemphigoid. I. Secretion of mucus and tears. Acta Ophthalmol (Copenh) 1974;52:266-81.

16. Norn MS, Kristensen EB. Benign mucous membrane pemphigoid. II. Cytology. Acta Ophthalmol (Copenh) 1974;52:282-90.

17. Van Joost T, Crone RA, Overdÿk AD. Ocular cicatricial pemphigoid associated with practolol therapy. Br J Dermatol 1976;94:447-50.

18. Duke-Elder S. Diseases of the outer eye. In: System of ophthalmology, vol 8. St Louis: CV Mosby, 1965:502-10.

19. Bean SF. Cicatricial pemphigoid: immunofluorescent studies. Arch Dermatol 1974;110:552-5.

20. Waltman SR, Yarian D. Circulating autoantibodies in ocular pemphigoid. Am J Ophthalmol 1974;77:891-4.

21. Franklin RM, Rice CD. Autoimmune diseases of the conjunctiva. In: O'Conner GR, editor. Immunologic diseases of the mucous membranes: pathology, diagnosis and treatment. New York: Masson, 1980:109-18. 